\title{
A Nonlinear Integral Model of Electron Devices for HB Circuit Analysis
}

\author{
Fabio Filicori, Giorgio Vannini, Student Member, IEEE, Vito A. Monaco, Senior Member, IEEE
}

\begin{abstract}
A technology-independent large-signal model of electron devices, the Nonlinear Integral Model (NIM), is proposed. It is rigorously derived from the Volterra series under basic assumptions valid for most types of electron devices and is suitable for Harmonic-Balance circuit analysis. Unlike other Volterra-based approaches, the validity of the NIM is not limited to weakly nonlinear operation. In particular, the proposed model allows the large-signal dynamic response of an electron device to be directly computed on the basis of data obtained either by conventional measurements or by physics-based numerical simulations. In this perspective, it provides a valuable tool for linking accurate device simulations based on carrier transport physics and Harmonic-Balance circuit analysis algorithms. Simulations and experimental results, which confirm the validity of the Nonlinear Integral Model, are also presented.
\end{abstract}

\section{INTRODUCTION}

$\mathrm{T}$ HE development of Monolithic Integrated Circuits for microwave applications [1]-[4] is strongly dependent on the availability of efficient and reliable CAD tools, since post-processing tuning is very difficult or practically impossible in MMIC's. At present, state-of-the-art CAD approaches [5]-[18], implemented in commercially available software packages, provide efficient tools for the performance evaluation and optimization of MMIC's. Electron device modeling plays a very important role in this context, since device performance prediction is the basic step for reliable circuit design. In this perspective, physics-based electron device models [19]-[25] are a valuable tool since they provide a direct link between physical parameters and electrical performance, by taking into account the basic equations of charge transport in semiconductors. In particular, the capability of predicting the influence of technology-related parameters on circuit performance is important for optimal "tailoring" of devices and for production yield optimization [17], [18]. Unfortunately, accurate numerical solution of the physical device equations is highly time-consuming since it involves two- or three-dimensional discretization of nonlinear partial derivative equations. Computational problems are particularly relevant in the case of nonlinear circuits,

Manuscript received July 25, 1991; revised February 10, 1992. This work was partly supported by the Italian National Research Council (CNR) and by research grants provided by Elettronica S.p.A.

F. Filicori is with Istituto di Ingegneria, Universitá di Ferrara, Via Scandiana 21, 44100 Ferrara, Italy and the CIOC Research Center of the National Research Council, Universitá di Bologna, Viale Risorgimento 2, 40136, Bologna, Italy.

G. Vannini and V. A. Monaco are with the Dipartimento di Elettronica, Informatica e Sistemistica, Universitá di Bologna, Viale Risorgimento 2 , 40136, Bologna, Italy.

IEEE Log Number 9200766 where many repeated evaluations of the device response are needed in the iterative procedures for nonlinear circuit analysis.

For the above reason numerical device analysis can only be carried out "off line" with respect to nonlinear circuit analysis, so that intermediate "behavioral" models are needed for the computationally efficient linking of numerical physics-based models with circuit analysis tools based on Harmonic-Balance (HB) techniques [6]-[8], [16]. To this end, "lumped" nonlinear equivalent circuits [3], [26]-[29], which have the advantage of being not only computationally efficient but also sufficiently accurate and reliable in most cases, can be derived from the results of numerical device simulations [21]. However, when largesignal operating conditions at microwave frequencies are considered, the identification of a nonlinear equivalent circuit is a relatively complex task. Owing to the presence of important nonlinear and parasitic phenomena, it can be rather difficult to identify the "lumped" equivalent circuit structure that best describes the electrical behavior of an electron device. Moreover, the "lumped" representation itself may not be sufficiently accurate, especially at very high frequencies, where it also may be necessary to consider "distributed" phenomena.

For the above reasons suitable approximation procedures, possibly based on numerical optimization, are needed to determine the parameter values which best "fit", an equivalent circuit to the electrical device characteristics. The need for special-purpose procedures to extract equivalent circuit parameters from numerical device simulations has the disadvantage of introducing an "indirect," complex link between physical device parameters and circuit performance. Moreover, the topology of equivalent circuits is strictly related to the particular structure of each type of electron device, so that the intrinsic flexibility of general purpose numerical device simulators is somehow lost. A better solution to the problem would be a general-purpose (i.e., purely mathematical [32], [33]) behavioral modeling approach that does not necessarily involve "lumped" approximations of the frequency-domain device response.

In this paper a technology-independent approach, which does not require complex parameter extraction procedures, is proposed for the circuit-oriented modeling of electron devices. It is based on the "Nonlinear Integral Model" (NIM) that is derived from the Volterra series by applying suitable consideration valid for most types of electron devices. In particular, the NIM allows the largesignal dynamic response of electron devices to be directly 
computed on the basis of bias- and frequency-dependent small-signal admittance parameters and dc characteristics; these can be either directly measured or computed by "off-line" physics-based device simulations.

The paper is structured as follows. In the next section, after briefly recalling the Volterra series, the Nonlinear Integral Model is derived. In Section III the identification of the NIM and its practical application to Harmonic-Balance circuit analysis is discussed. Finally, some practical results, which confirm the validity of the modeling approach proposed, are presented.

\section{The Nonlinear Integral Model}

The difficulties related to circuit-oriented large-signal modeling of microwave electron devices are mainly due to the simultaneous presence of nonlinear and dynamic phenomena. By considering, for simplicity, a single port device", the terms "nonlinear" and "dynamic" imply that the current $i(t)$ at any time instant $t$ is nonlinearly dependent not only on the applied voltage $v(t)$ at the same instant, but also on past values $v(\tau)$ in the time interval $t-\tau_{m} \leq \tau<t$, where $\tau_{m}$ represents the practically finite duration of the "memory" effects associated with dynamic phenomena in the active device (e.g., charge-storage effects, carrier transit times, etc.). The relation between the parameter $\tau_{m}$ and the high-frequency, largesignal performance of an electron device will be discussed in the following.

The most general, rigorous modeling approach for systems characterized by nonlinear dynamic phenomena is the well-known Volterra series [34]-[38]. This approach is based on the description of the nonlinear dynamic effects by means of multi-dimensional convolution integrals which can be considered to be a generalization of the onedimensional convolution integral for dynamic linear systems; the nonlinear effects, in particular, are described by means of a multi-dimensional integral polynomial representation which is analogous to the Taylor series expansion for purely algebraic nonlinear systems. On these bases the current/voltage response of a single-port device under large-signal operation can be expressed in the time domain by means of the Volterra series:

$$
\begin{aligned}
i(t)= & \int_{t-\tau_{m}}^{t} h_{1}\left(t-\tau_{1}\right) v\left(\tau_{1}\right) d \tau_{1} \\
& +\iint_{t-\tau_{m}}^{t} h_{2}\left(t-\tau_{1}, t-\tau_{2}\right) v\left(\tau_{1}\right) v\left(\tau_{2}\right) d \tau_{1} d \tau_{2} \\
& +\cdots \\
= & \sum_{n=1}^{\infty} \int \cdots \int_{t-\tau_{m}}^{t} h_{n}\left(t-\tau_{1}, \cdots, t-\tau_{n}\right) \\
& \cdot v\left(\tau_{1}\right) \cdots v\left(\tau_{n}\right) d \tau_{1} \cdots d \tau_{n}
\end{aligned}
$$

'The following mathematical development can also be made for multiport devices as shown in the Appendix. where the integration interval is limited to the practically finite duration $\tau_{m}$ of the "memory" effects in the device. The functions $h_{n}\left(\tau_{1}, \cdots, \tau_{n}\right)$, which are known as Volterra kernels of order $n$, represent multi-dimensional impulse responses which describe the dynamic nonlinear characteristics of the device.

The Volterra series can be efficiently used only when weakly nonlinear operation is considered since, in such conditions, the finite number $N$ of kernels $h_{n}$ which are required in order to have a good approximation of series (1) is small (typically $N=3-5$ ) [36]-[40]. When the amplitude of the signals becomes large with respect to the nonlinearities of the device, the number $N$ of kernels which must be taken into account substantially increases and the Volterra modeling approach becomes impractical. Moreover, at microwave frequencies, suitable instrumentation for the measurement of the kernels [32], [39], [40] is still lacking; this limits the practical usefulness of the Volterra series. $^{2}$

In order to improve the convergence properties of the Volterra series in the case of electron device modeling, it is convenient to define the "dynamic deviations":

$$
e\left(\tau_{i}, t\right)=v\left(\tau_{i}\right)-v(t) \quad i=1,2, \cdots
$$

between the voltages $v\left(\tau_{i}\right)$ at the time instants $\tau_{i}<t$ and the voltage $v(t)$. In particular, by using (2), (1) leads to the Nonlinear Integral Series ${ }^{3}$ :

$$
\begin{aligned}
i(t)= & F_{\mathrm{DC}}\{v(t)\}+\sum_{k=1}^{\infty} \int \cdots \int_{t-\tau_{m}}^{t} \\
& \cdot g_{k}\left\{v(t), t-\tau_{1}, \cdots, t-\tau_{k}\right\} e\left(\tau_{1}, t\right) \\
& \cdots e\left(\tau_{k}, t\right) d \tau_{1} \cdots d \tau_{k}
\end{aligned}
$$

where the functions $F_{\mathrm{DC}}$ and $g_{k}$ are related to the Volterra kernels $h_{n}$ (see Appendix) by

$$
\begin{aligned}
F_{\mathrm{DC}}\{v(t)\}= & \sum_{n=1}^{\infty} v^{n}(t) \int \cdots \int_{t-\tau_{m}}^{t} \\
& \cdot h_{n}\left(t-\tau_{1}, \cdots, t-\tau_{n}\right) d \tau_{1} \cdots d \tau_{n}
\end{aligned}
$$

and:

$$
\begin{aligned}
g_{k}\left\{v(t), t-\tau_{1}, \cdots, t-\tau_{k}\right\} & \\
= & \sum_{n=k}^{\infty} v^{(n-k)}(t)\left(\begin{array}{l}
n \\
k
\end{array}\right) \int \cdots \int_{t-\tau_{m}}^{t} \\
& \cdot h_{n}\left(t-\tau_{1}, \cdots, t-\tau_{n}\right) d \tau_{k+1} \cdots d \tau_{n}
\end{aligned}
$$

${ }^{2}$ In spite of this drawback, the Volterra series has been used for microwave circuit design, by means of analytical or numerical calculation of the kernels [32], [36], [37]

${ }^{3}$ The mathematical demonstration is given in the Appendix for the more general case of a two-pont device. 
with:

$$
\left(\begin{array}{l}
n \\
k
\end{array}\right)=\frac{n !}{(n-k) ! k !} .
$$

The nonlinear function $F_{\mathrm{DC}}$ in (3), coincides with the static characteristic of the device since, in dc steady-state operation, the terms $e\left(\tau_{i}, t\right)=v\left(\tau_{i}\right)-v(t)$ in the integrals are identically zero. So, the strictly dynamic phenomena are described by the multi-dimensional convolution integrals in terms of the nonlinearly voltage-controlled impulse-response $g_{k}$ and the voltage dynamic deviations $e\left(\tau_{i}, t\right)$.

Equation (3) provides fast convergence when the dynamic deviations $e\left(\tau_{i}, t\right)$ are small enough since, in such conditions, the multi-dimensional integrals quickly decrease; this happens even in the presence of large-voltage amplitudes, provided that the practically finite duration $\tau_{m}$ of memory effects is sufficiently small. This condition, as will be shown in the following, is satisfied for the most common types of electron devices in typical analog applications. In particular, even under large-signal and strongly nonlinear operating conditions, the dynamic deviations $e\left(\tau_{i}, t\right)$ are almost always small enough to make negligible the integrals of dimension greater than one in (3); this leads to the following equation:

$$
\begin{aligned}
i(t)= & F_{\mathrm{DC}}\{v(t)\}+\int_{t-\tau_{m}}^{t} g_{1}\{v(t), t-\tau\} \\
& \cdot[v(\tau)-v(t)] d \tau
\end{aligned}
$$

which defines the Nonlinear Integral Model and consists of a single-fold convolution (nonlinearly controlled by the instantaneous voltage $v(t)$ ) with respect to the voltage dynamic deviations $e(\tau, t)=v(\tau)-v(t)$.

In order to understand why the voltage-controlled expression (6) can correctly describe even the strongly nonlinear response of an electron device, a few considerations, besides the experimental and simulated results given in Section IV, are needed. To this end, it should be taken into account that any spectral component $V(\omega)$ of the voltage $v(t)$ gives a contribution to the dynamic deviation $v(\tau)-v(t)$ which can be expressed in the form $\operatorname{Re}\left\{V(\omega)\left[e^{j \omega \tau}-e^{j \omega t}\right]\right\}$; clearly, this contribution may have a small amplitude, even for a large amplitude of $V(\omega)$, provided that the "short-term" memory condition:

$$
\max (t-\tau)=\tau_{m} \ll \frac{1}{\omega}
$$

holds for any angular frequency $\omega$ where large-amplitude voltage spectral components (i.e., large enough to stimulate nonlinear behavior) are present. Thus, it can be said that the NIM (6) is valid provided that $\tau_{m} \ll 1 / B_{\mathrm{LS}}$, where $B_{\mathrm{LS}}$ represents the large-signal bandwidth of the applied voltage (that is, the maximum angular frequency where spectral components $V(\omega)$ are present with an amplitude large enough to directly stimulate nonlinear behavior in the electron device). It should be noted that sim- ply considering the maximum angular frequency $\omega_{\max }$ of $v(t)$ (i.e., the maximum frequency where non negligible spectral components can be found) would have been more restrictive than strictly necessary. In fact, in order to guarantee the validity of (6), inequality (7) must be satisfied only for large-amplitude spectral components; no constraint needs to be imposed on those spectral components whose amplitude is not large enough to autonomously generate nonlinear behavior ${ }^{4}$, although the same amplitude is not negligible for accurate circuit analysis (owing to important reactive phenomena).

Under typical large-signal operating conditions, the amplitude of the higher-order signal spectra components decreases quite quickly with increasing frequency, so that $B_{\mathrm{LS}}$ does not usually exceed the second- or third-order harmonic component of the fundamental frequency; consequently, for typical applications of electron devices, $B_{\mathrm{LS}}$ will not generally be much higher (i.e., it has the same order of magnitude) than the maximum operating frequency given by the manufacturer.

The "short-term" memory condition $\tau_{m} \ll 1 / B_{\mathrm{LS}}$ is verified for most types of electron devices when described in a voltage-controlled form. This property, which can be intuitively explained by considering that nonlinear dynamic effects in electron devices are mainly associated with voltage-controlled charge-storage phenomena or very short transit times, is also confirmed by experimental evidence. For instance, the duration of the current transient responses to voltage pulses applied either to the source or gate terminals of GaAs MESFET's, is generally much smaller than the inverse of the typical operating frequency given by the manufacturer (see for instance Figs. 1 and 2 where the gate and drain current responses to an ideal voltage pulse applied to the gate are shown for the devices in Table I).

Similar results can be found for other electron devices (e.g., bipolar transistors, junction diodes, etc.) provided a voltage-controlled description is used and the intrinsic device (i.e., the "active" part of the device) is not subject to dominant parasitic effects; this is generally true for devices used in monolithic circuits or discrete devices in "bare-chip" form. In the case of packaged discrete devices, the package parasitics may slow down the impulse response of the device and make the assumption of shortterm memory not completely acceptable for very high operating frequencies; under such conditions good accuracy can still be achieved with the NIM provided that conventional techniques for "deembedding" from the linear parasitics are used [3].

The above discussion of short-term memory effects should not lead to doubts about the capability of the NIM to take into account reactive effects in an electron device under typical high-frequency nonlinear operation. In fact, although the current responses to ideal voltage pulses are

\footnotetext{
"It must be emphasized that (6) is intrinsically "exact" in small-signa operation, since, under such conditions, it reduces to the linear convolution
} integral. 


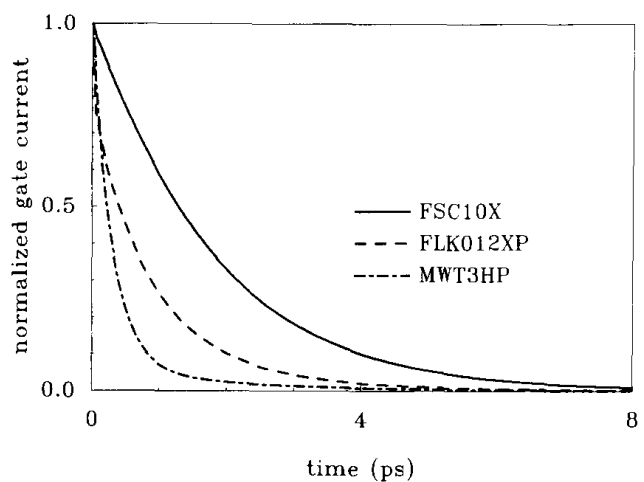

Fig. 1. Gate current responses to a gate voltage pulse for the GaAs MESFETs in Table I. The responses have been normalized to their peak value in order to make the comparison between different devices easier.

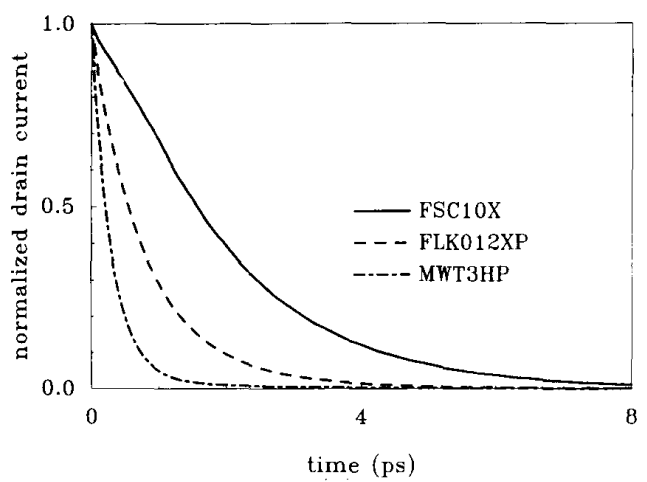

Fig. 2. Drain current responses to a gate voltage pulse for the GaAs MESFETs in Table I. The responses have been normalized to their peak value in order to make the comparison between different devices easier.

TABLE I

Typical Operating Band and Current-Gain Cutoff Frequency $f_{T}$ Given by the ManUfacturer for GaAS MESFET'S

\begin{tabular}{|c|c|c|c|}
\hline Device & Operating Band & Bias & $f_{T}$ \\
\hline $\begin{array}{l}\text { FSC10X } \\
\text { (Fujitsu) }\end{array}$ & $C(4-8 \mathrm{GHz})$ & $\begin{array}{l}V_{D S}=3 \mathrm{~V} \\
I_{\mathrm{DS}}=10 \mathrm{~mA}\end{array}$ & $11.5 \mathrm{GHz}$ \\
\hline $\begin{array}{l}\text { FLK012XP } \\
\text { (Fujitsu) }\end{array}$ & $X-K_{u}(8-18 \mathrm{GHz})$ & $\begin{array}{l}V_{\mathrm{DS}}=10 \mathrm{~V} \\
I_{\mathrm{DS}}=36 \mathrm{~mA}\end{array}$ & $16.1 \mathrm{GHz}$ \\
\hline $\begin{array}{l}\text { MWT3HP } \\
\quad \text { (Microwave Tech.) }\end{array}$ & $K(18-26 \mathrm{GHz})$ & $\begin{array}{l}V_{\mathrm{DS}}=6 \mathrm{~V} \\
l_{\mathrm{DS}}=50 \mathrm{~mA}\end{array}$ & $19.3 \mathrm{GHz}$ \\
\hline
\end{tabular}

very fast, this does not mean that reactive effects are somehow neglected or underestimated; roughly speaking, reactive effects are related not only to the duration of the pulse responses, but also to the area of those responses which can be very large due to the extremely high displacement currents corresponding to ideal voltage pulses.

Equation (6) can be directly used in the case of multiport devices, as shown in the Appendix, by interpreting the functions $F_{\mathrm{DC}}, g_{1}$ and $i(t), v(t)$ respectively as matrices and vectors of suitable dimensions.

The Nonlinear Integral Model can also take into account certain other phenomena which do not belong to the class of "short-memory" effects, such as the low-frequency dispersion of electrical characteristics in GaAs electron devices. In particular, under small-signal operation, the NIM is intrinsically exact whatever frequency of operation is considered; this applies also for low-frequency dispersion phenomena. As far as the direct influence of these phenomena on the large-signal performance of an electron device is concerned, theoretical considerations and experimental results confirming the validity of the NIM are given in [41].

\section{The Nonlinear Integral Model for HB Circuit Analysis}

The Nonlinear Integral Model described by (6) could be used for time-domain circuit analysis by applying conventional numerical integration techniques [8]. However, when microwave applications are considered, circuit analysis is more conveniently carried out in the frequency domain by means of Harmonic-Balance techniques [6][8], [16]. It can easily be shown that (6) can be written, by applying well known properties of the Fourier transform, in a new form which is particularly suitable for HB circuit analysis. To this end, by considering the Fourier transforms $V(\omega)$ of $v(t)$, and $G_{1}\{v(t), \omega\}$ of $g_{1}\{v(t), \tau\}$ :

$$
\begin{gathered}
V(\omega)=\int_{0}^{\tau_{m}} v(t) e^{-j \omega t} d t \\
G_{1}\{v(t), \omega\}=\int_{0}^{\tau_{m}} g_{1}\{v(t), \tau\} e^{-j \omega \tau} d \tau
\end{gathered}
$$

and developing the product inside the integral in (6), the latter can be written in the form:

$$
\begin{aligned}
i(t)= & F_{\mathrm{DC}}\{v(t)\}+\frac{1}{2 \pi} \int_{-\infty}^{+\infty} G_{1}\{v(t), \omega\} V(\omega) e^{j \omega t} d \omega \\
& -\frac{1}{2 \pi} \int_{-\infty}^{+\infty} G_{1}\{v(t), 0\} V(\omega) e^{j \omega t} d \omega
\end{aligned}
$$

where the time-domain convolution has been expressed as the inverse Fourier transform of the product $G_{1}\{v(t)$, $\omega\} V(\omega)$.

By taking into account (9) and letting:

$$
\begin{aligned}
\tilde{Y}\{v(t), \omega\} & =G_{1}\{v(t), \omega\}-G_{1}\{v(t), 0\} \\
& =\int_{0}^{\tau_{m}} g_{1}\{v(t), \tau\}\left[e^{-j \omega \tau}-1\right] d \tau
\end{aligned}
$$

(10) becomes:

$$
i(t)=F_{\mathrm{DC}}\{v(t)\}+\frac{1}{2 \pi} \int_{-\infty}^{+\infty} \tilde{Y}\{v(t), \omega\} V(\omega) e^{j \omega t} d \omega
$$

When we are concerned with Harmonic-Balance circuit analysis, discrete-spectrum voltages and currents are considered which can be expressed by means of the well- 
known Fourier representations:

$$
i(t)=\sum_{p=-P}^{+P} I_{p} e^{j \omega_{p} t}, \quad v(t)=\sum_{p=-P}^{+P} V_{p} e^{j \omega_{p} t}
$$

where $P$ represents the practically finite number of harmonic components, while $V_{p}$ and $V_{-p}=V_{p}^{*}$ (and, analogously $I_{p}$ and $I_{-p}=I_{p}^{*}$ ) are complex conjugate phasors at the angular frequencies $\omega_{p}$ and $\omega_{-p}=-\omega_{p}$ (with $\omega_{0}=$ $0)$.

Under such conditions, (12) can be more conveniently expressed in the Harmonic-Balance oriented form ${ }^{5}$ :

$$
\begin{aligned}
i(t) & =\sum_{p=-P}^{+P} I_{p} e^{j \omega_{p} t} \\
& =F_{\mathrm{DC}}\{v(t)\}+\sum_{p=-P}^{+P} \tilde{Y}\left\{v(t), \omega_{p}\right\} V_{p} e^{j \omega_{p} t}
\end{aligned}
$$

The term $\tilde{Y}$ in (14) represents a voltage-dependent " $d y$ namic" admittance which describes the purely dynamic phenomena in the device, since for $\omega=0$, according to (11), $\tilde{Y}\{v(t), \omega\}=0$. The dynamic admittance $\tilde{Y}$ is nonlinearly dependent only on the instantaneous voltage $v(t)$; this is justified by the hypothesis of short-term memory and is consistent with similar assumptions used in the derivation of some nonlinear equivalent circuits [3], [26].

The dynamic and nonlinearly voltage-controlled admittance $\tilde{Y}\{v(t), \omega\}$ is related to the classical bias-dependent small-signal admittance $Y$ by a very simple relation. In fact, by considering a small sinusoidal "test", voltage superimposed on a given dc bias voltage $V_{B}$, linearization of (14) with respect to the small signal, leads to the following expression:

$$
\left(\frac{d I_{p}}{d V_{p}}\right)_{V_{B}} \doteq Y\left[V_{B}, \omega_{p}\right]=g_{\mathrm{DC}}\left[V_{B}\right]+\tilde{Y}\left[V_{B}, \omega_{p}\right]
$$

where $g_{\mathrm{DC}}=\left(d F_{\mathrm{DC}} / d v\right)_{V_{B}}$ is the dc differential conductance of the device.

Equation (15) shows that the identification of the mathematical model proposed here is really straightforward as it can be carried out directly, without requiring numerical fitting procedures, on the basis of dc characteristics and small-signal admittance parameters computed (or measured) for several dc bias conditions and operating frequencies over the range of interest. On the basis of (15), the nonlinearly voltage-controlled dynamic admittance $\tilde{Y}\left\{v, \omega_{p}\right\}$ can be found by computing or measuring $Y$ and $g_{\text {DC }}$ parameters for each value of $v=V_{B}$ :

$$
\begin{gathered}
\tilde{Y}\left\{v, \omega_{p}\right\}=\tilde{Y}\left[V_{B}, \omega_{p}\right]=Y\left[V_{B}, \omega_{p}\right]-g_{\mathrm{DC}}\left[V_{B}\right] \\
\text { with } v=V_{B} .
\end{gathered}
$$

\footnotetext{
${ }^{5}$ As already stated for the Nonlinear Integral Model (6), (14) can be directly used in the case of multi-port devices by interpreting the functions $F_{\mathrm{DC}}, \tilde{Y}$ and $i(t), v(t), V_{p}$, respectively as matrices and vectors of suitable dimensions.
}

This type of characterization can easily be obtained when a suitable tool for the physics-based simulation of semiconductor devices [19]-[21] is available. The identification of model (14) can be carried out also on the basis of experimental data (e.g., bias-dependent $S$-parameters and $\mathrm{dc}$ characteristics). The availability of automatic network analyzers and automatic dc source/monitor equipment makes this task relatively simple.

Once the dc characteristic $F_{\mathrm{DC}}$ and the small-signal admittance $Y$ have been computed (or measured), (14) and (16) can easily be used, by applying suitable interpolation techniques ${ }^{6}$, for large-signal performance prediction in the framework of Harmonic-Balance circuit analyses.

The simplicity of identification which characterizes the Nonlinear Integral Model, makes it very suitable for use with physical simulators in order to obtain a computationally efficient link between accurate physics-based device simulations and HB circuit analysis, Moreover, in comparison with equivalent circuit models, it presents several advantages. In small-signal operation, the NIM reduces to the small-signal admittance parameters directly computed (or measured). Thus, under small-signal conditions, the model proposed does not require any approximation of the frequency response of the device, and is intrinsically exact ${ }^{7}$. It must be observed that when a "lumped" equivalent circuit model is adopted the frequency response of the electron device is necessarily approximated. In particular, this is one of the reasons for the need of numerical fitting procedures in parameter extraction.

Moreover, the model proposed is particularly suitable when the designer is concerned with computing the sensitivity $S_{P}^{B}=\partial B / \partial P$ of a circuit performance $B$ with respect to a process-dependent parameter $P$. To this end, it must be emphasized that the NIM allows the closed-form expression (14) to be used for computation of the largesignal response of an electron device, directly in terms of dc characteristics and small-signal admittance parameters. Thus the computation of the sensitivity $S_{P}^{B}$ requires only knowledge of the sensitivities of the dc characteristics and $Y$-parameters with respect to the process-dependent parameter $P$. These sensitivities can be efficiently obtained when suitable tools for physics-based simulation of active devices are available [42].

\footnotetext{
'Since, in practice, only a "discrete" (i.e., discrete in the space of the controlling voltages) electrical characterization of the device can be carried out by means of numerical device simulations or measurements, interpolation techniques are required to compute the dc characteristic $F_{\mathrm{DC}}\{v(t)\}$ and the dynamic admittance $\tilde{Y}\left\{v(t), \omega_{p}\right\}$ for each value of the voltage $v(t)$. When using Harmonic-Balance analysis, the choice of the interpolation method for the NIM is not very critical, in the sense that particular properties of regularity (differentiability) are not required. This is due to the "smoothing" properties of the Fourier Transform algorithms which are used to express, in the frequency domain, the time-domain response of active devices. Thus, conventional piece-wise linear interpolation techniques can be adopted. However, the choice of a higher-order "smooth", interpolation method can considerably improve the accuracy of the NIM, without the need for increasing the refinement of the "discrete" numerical (or experimental) characterization.

${ }^{7}$ Obviously apart from possible numerical (or measurement) errors in the computation (or measurement) of small-signal admittance parameters.
} 


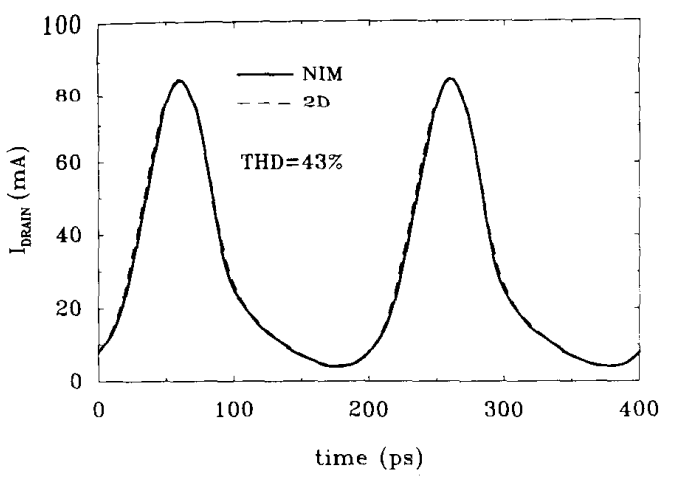

Fig. 3. Plots of the time-varying drain current in a GaAs MESFET amplifier with sinusoidal input at $5 \mathrm{GHz}\left(P_{\mathrm{IN}}=10 \mathrm{dBm}, V_{\mathrm{GSO}}=-2 \mathrm{~V}, V_{\mathrm{DSO}}\right.$ $=4 \mathrm{~V}$ ). The large-signal performance predicted by the NIM (-) is in good agreement with 2D physics-based numerical device simulation (--). The Total Harmonic Distortion is $43 \%$.

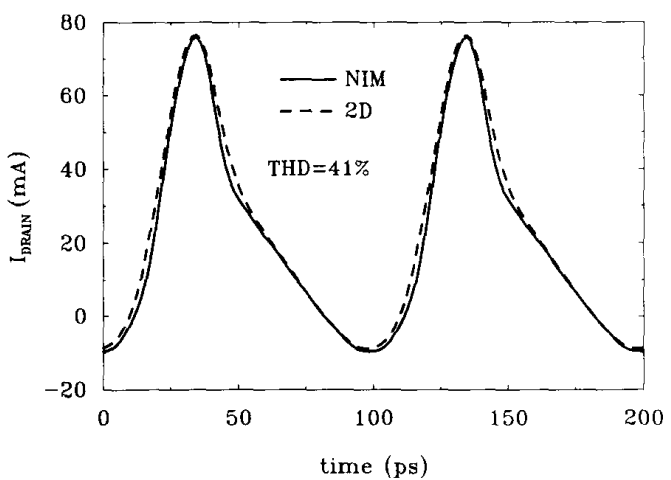

Fig. 4. Plots of the time-varying drain current in a GaAs MESFET amplifier with sinusoidal input at $10 \mathrm{GHz}\left(P_{\mathrm{IN}}=13 \mathrm{dBm}, V_{\mathrm{GSO}}=-3 \mathrm{~V}\right.$, $\left.V_{D S 0}=4 \mathrm{~V}\right)$. The large-signal performance predicted by the NIM $(-$ is in good agreement with 2D physics-based numerical device simulation $(--)$. The Total Harmonic Distortion is $41 \%$.

When an equivalent circuit model is adopted to predict the large-signal response of an electron device, the computation of the sensitivity $S_{P}^{B}$ is not a simple task since it requires calculating the sensitivities $s_{P}^{C}=\partial C / \partial P$ of model parameters $C$ with respect to the process-dependent parameter $P$. Such a computation can be quite cumbersome, since the determination of the parameters $C$ of the equivalent circuit is carried out by means of numerical fitting procedures, and a direct link between such parameters and process-dependent variables is not available.

\section{Simulations and Experimental Results}

As has been stated, the NIM represents a useful approach to electron device modeling for circuit analysis purposes, since it provides a direct link between conventional measurements or accurate device simulations based on carrier transport physics and those based on HarmonicBalance circuit analysis. In order to verify the validity of the approach proposed, a comparison of the results ob-

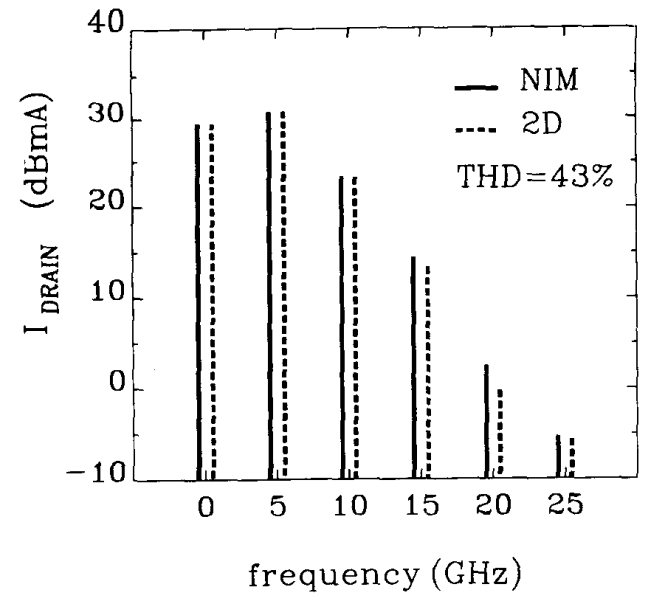

Fig. 5. Plots of the current spectra associated with the time-varying waveforms in Fig. 3. The results obtained by the NIM (__ ) are in good agreement with those obtained by means of $2 \mathrm{D}$ physics-based numerical device simulation (---)

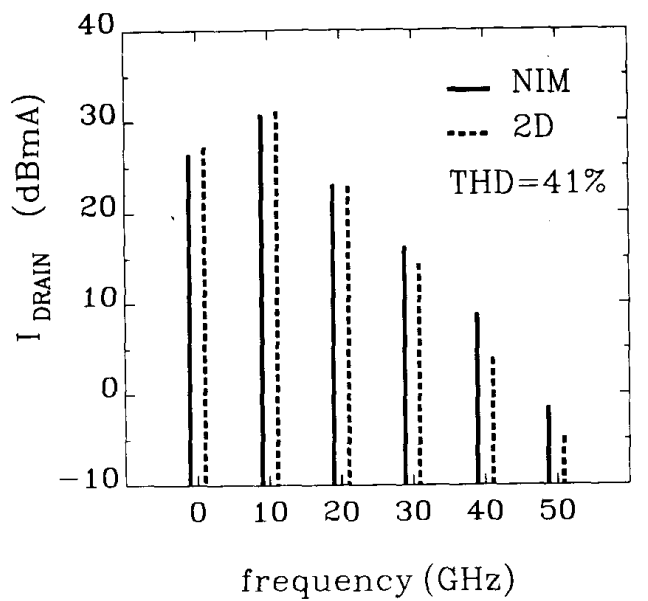

Fig 6. Plots of the current spectra associated with the time-varying waveforms in Fig. 4. The results obtained by the NIM (-) are in good agreement with those obtained by means of $2 \mathrm{D}$ physics-based numerical device simulation (---).

tained in circuit analysis by means of the NIM and those obtained by means of physics-based simulations has been carried out.

In particular, a medium power $X$-band GaAs MESFET with $0.4 \mu \mathrm{m}$ gate length, grown by Molecular Beam Epitaxy, has been considered. Physics-based numerical simulations [20], [21] have been carried out in order to obtain a complete dc characterization and the small-signal admittance parameters of the MESFET for several bias points (about 500 in the $V_{G S O}, V_{\mathrm{DSO} O}$ space) and for $10 \mathrm{har}$ monic frequencies (with fundamental at 5 and $10 \mathrm{GHz}$ ). On the basis of this characterization and by means of (14), obviously considered as a matrix representation, the HB analysis of a class- $\mathrm{AB}$ amplifier at the frequencies of 5 and $10 \mathrm{GHz}$ loaded with a $50 \Omega$ impedance has been carried out. The nonlinearly voltage-controlled dynamic admittance $\tilde{Y}\left\{v_{\mathrm{GS}}(t), v_{\mathrm{DS}}(t), \omega\right\}$ which describes the purely 


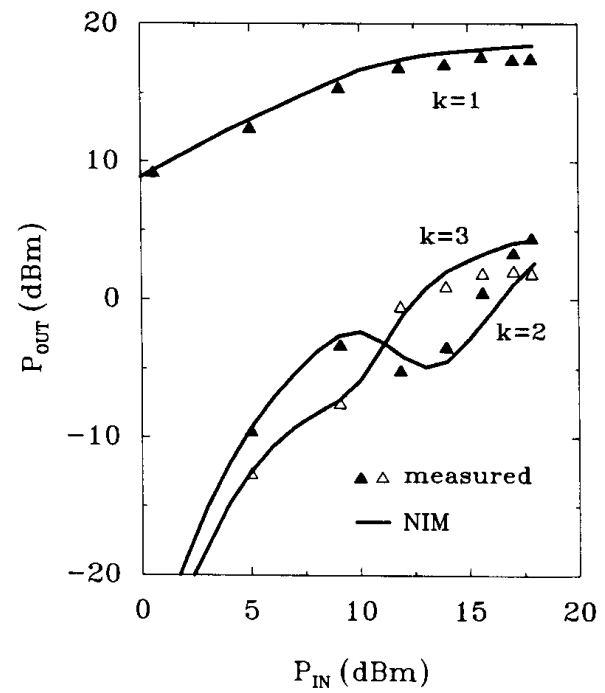

Fig. 7. Plots of output powers associated with different order harmonics $(k=1,2,3)$ vs input power $P_{\mathrm{IN}}$ for a $K_{u}$-band GaAs MESFET amplifier $\left(V_{\mathrm{GSO}}=-0.4 \mathrm{~V}, V_{\mathrm{DSO}}=4 \mathrm{~V}\right)$ with sinusoidal input $\left(f_{0}=8 \mathrm{GHz}\right)$. Experimental results $(\boldsymbol{\Lambda}, \Delta)$ are in good agreement with those predicted by the NIM (- - $)$

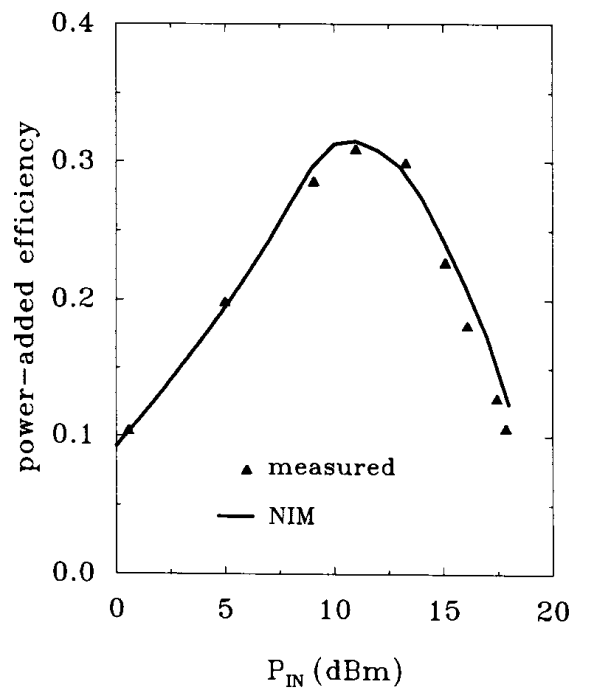

Fig. 8. Plot of the power-added efficiency vs input power $P_{\text {IN }}$ for a $K$ band GaAs MESFET amplifier $\left(V_{\mathrm{GS} 0}=-0.4 \mathrm{~V}, V_{\mathrm{DS} 0}=4 \mathrm{~V}\right)$ with sinusoidal input $\left(f_{0}=8 \mathrm{GHz}\right)$. Experimental results $(\Delta)$ are in good agreement with those predicted by the NIM ( - .

dynamic phenomena in the device, was computed according to (16), for each pair of instantaneous voltage $v_{\mathrm{GS}}(t)$, $v_{\mathrm{DS}}(t)$, occurring in the HB analysis, by using piece-wise linear interpolation techniques in the two-dimensional $V_{\mathrm{GS} 0}, V_{\mathrm{DS} 0}$ space.

Figs. 3 to 6 show the time-varying waveforms of the drain current $i_{D}(t)$ and the associated spectra, obtained by the HB circuit analysis for the above-mentioned amplifier. In the same figures, the results obtained by car- rying out the corresponding analyses by means of the physics-based full two-dimensional simulator [20], [21], at the cost of a very long computation time, are also drawn. The good agreement between circuit analyses carried out on the basis of the NIM and the large-signal prediction obtained for the same circuit by means of an accurate physics-based numerical simulator, confirms that the Nonlinear Integral Model can be used as an accurate, computationally efficient tool for linking numerical device simulators with $\mathrm{HB}$ analysis algorithms.

An experimental validation has also been carried out for the Nonlinear Integral Model. In particular, validation tests have been carried out at the frequency of $8 \mathrm{GHz}$ on a large-signal amplifier using a $K_{u}$ band GaAs MESFET. Figs. 7 and 8 show, respectively, plots of the output power associated with different order harmonics and the poweradded efficiency as functions of the input power. Also in this case, the agreement between the results provided by the model proposed and large-signal measurements confirms the accuracy of the NIM.

\section{CONCLUSION}

A new technology-independent modeling approach for the large-signal performance prediction of electron devices has been proposed. The Nonlinear Integral Model has been derived from the Volterra series through suitable modifications, so that fast convergence can be achieved under the assumption, valid for most types of electron devices, of only "short-term" memory effects. The model, which can be directly used for Harmonic-Balance circuit analysis, enables the large-signal response of electron devices to be directly computed by means of a closed-form expression based on dc characteristics and small-signal $Y$ parameters computed (or measured) for different bias conditions over the frequency range of interest. This property makes the Nonlinear Integral Model particularly convenient for the computationally efficient linking of numerical device simulators with Harmonic-Balance circuit analysis algorithms. Moreover, the computation of the sensitivities of circuit performance with respect to process-dependent parameters is a relatively easy task when using the Nonlinear Integral Model.

The comparison of both accurate physics-based device simulations and performance measurements with the results provided by Harmonic-Balance circuit analysis using the Nonlinear Integral Model has confirmed the validity of this mathematical modeling approach.

\section{APPENDIX}

\section{Derivation of the Nonlinear Integral Model from the Volterra Series}

Let us consider the Volterra series (1) in its bivariate ${ }^{8}$ form [34], [38]:

${ }^{8}$ The following demonstration can be directly extended to devices with any number of ports. 


$$
\begin{aligned}
i(t)= & \sum_{k=0}^{\infty} \sum_{n=0}^{\infty}{ }^{(k)} \int_{t-\tau_{m}}^{t} \int_{t-\tau_{m}}^{(n)}{ }^{t} \\
& \cdot h_{k n}\left(t-\bar{\tau}_{1}, \cdots, t-\bar{\tau}_{k} ; t-\hat{\tau}_{1}, \cdots, t-\hat{\tau}_{n}\right) \\
& \cdot \bar{v}\left(\bar{\tau}_{1}\right) \cdots \bar{v}\left(\bar{\tau}_{k}\right) \hat{v}\left(\hat{\tau}_{1}\right) \cdots \hat{v}\left(\hat{\tau}_{n}\right) d \bar{\tau}_{1} \\
& \cdots d \bar{\tau}_{k} d \hat{\tau}_{1} \cdots d \hat{\tau}_{n}
\end{aligned}
$$

where the superscripts $(k),(n)$ denote the dimensions of the integrals ${ }^{9} ; \bar{v}, \hat{v}$ are the voltages at the two ports of the device and $i$ any of the associated currents.

The multi-dimensional impulse responses $h_{k n}$ represent the Volterra kernels which are symmetric functions of their arguments [35], in the sense that the sequence of the arguments can be interchanged in $h_{k n}$ without modifying the results.

In order to derive the Nonlinear Integral Model the voltages $\bar{v}\left(\bar{\tau}_{k}\right), \hat{v}\left(\hat{\tau}_{n}\right)$ must be expressed, according to the considerations made in Section II, in terms of the dynamic deviations $\bar{e}\left(\bar{\tau}_{k}, t\right), \hat{e}\left(\hat{\tau}_{n}, t\right)$ :

$$
\begin{array}{ll}
\bar{v}\left(\bar{\tau}_{k}\right)=\bar{v}(t)+\bar{e}\left(\bar{\tau}_{k}, t\right) & k=1,2, \cdots \\
\hat{v}\left(\hat{\tau}_{n}\right)=\hat{v}(t)+\hat{e}\left(\hat{\tau}_{n}, t\right) & n=1,2, \cdots
\end{array}
$$

By substituting (18) into (17) and applying the symmetry property of the kernels to collect in a single term all the integrals of the same dimension which differ only in the position of the integration variables, we can write:

$$
\begin{aligned}
i(t)= & \sum_{k=0}^{\infty} \sum_{n=0}^{\infty} \sum_{p=0}^{k} \sum_{q=0}^{n} \bar{v}^{(k-p)}(t)\left(\begin{array}{l}
k \\
p
\end{array}\right) \hat{v}^{(n-q)}(t)\left(\begin{array}{l}
n \\
q
\end{array}\right) \\
& \cdot \int_{t-\tau_{m}}^{(k)} \int_{t-\tau_{m}}^{t} h_{k n}\left(t-\bar{\tau}_{1}, \cdots, t-\bar{\tau}_{k}\right. \\
& \left.t-\hat{\tau}_{1}, \cdots, t-\hat{\tau}_{n}\right) \bar{e}\left(\bar{\tau}_{1}, t\right) \cdots \bar{e}\left(\bar{\tau}_{p}, t\right) \\
& \cdot \hat{e}\left(\hat{\tau}_{1}, t\right) \cdots \hat{e}\left(\hat{\tau}_{q}, t\right) d \bar{\tau}_{1} \cdots d \bar{\tau}_{k} d \hat{\tau}_{1} \cdots d \hat{\tau}_{n}
\end{aligned}
$$

where:

$$
\left(\begin{array}{l}
k \\
p
\end{array}\right)=\frac{k !}{(k-p) ! k !}, \quad\left(\begin{array}{l}
n \\
q
\end{array}\right)=\frac{n !}{(n-q) ! q !}
$$

In (19) the term corresponding to $(p, q)=(0,0)$ depends only on the instantaneous values of the voltages $\bar{v}, \hat{v}$, and represents the dc characteristic:

$$
\begin{aligned}
F_{\mathrm{DC}}\{\bar{v}(t), \hat{v}(t)\}= & \sum_{k=0}^{\infty} \sum_{n=0}^{\infty} \bar{v}^{k}(t) \hat{v}^{n}(t) \\
& \cdot \int_{t-\tau_{m}}^{{ }^{(k)}} \int_{t-\tau_{m}}^{t} h_{k n}\left(t-\bar{\tau}_{1}, \cdots,\right. \\
& \left.t-\bar{\tau}_{k} ; t-\hat{\tau}_{1}, \cdots, t-\hat{\tau}_{n}\right) \\
& \cdot d \bar{\tau}_{1} \cdots d \bar{\tau}_{k} d \hat{\tau}_{1} \cdots d \hat{\tau}_{n}
\end{aligned}
$$

${ }^{9}$ The term corresponding to $(k, n)=(0,0)$ does not need to be taken into account when electron device modeling is concerned, since an electron device under "zero-voltage" excitations has "zero-current" response.
By inverting the order of the summations in (19), and taking into account that according to the same equation there are no terms with index $p>k, q>n$, the following expression can be obtained:

$$
\begin{aligned}
i(t)= & F_{\mathrm{DC}}\{\bar{v}(t), \hat{v}(t)\} \\
& +\sum_{\substack{p=0 \\
(p, q) \neq(0,0)}}^{\infty} \sum_{\substack{q=0 \\
(k)}}^{\infty} \sum_{k=p}^{\infty} \sum_{n=q}^{\infty} \bar{v}^{(k-p)}(t)\left(\begin{array}{l}
k \\
p
\end{array}\right) \hat{v}^{(n-q)}(t)\left(\begin{array}{l}
n \\
q
\end{array}\right) \\
& \cdot \int_{t-\tau_{m}}^{(n)} \int_{t-\tau_{m}}^{t} h_{k n}\left(t-\bar{\tau}_{1}, \cdots, t-\bar{\tau}_{k}\right. \\
& \left.t-\hat{\tau}_{1}, \cdots, t-\hat{\tau}_{n}\right) \bar{e}\left(\bar{\tau}_{1}, t\right) \\
& \cdots \cdot \bar{e}\left(\bar{\tau}_{p}, t\right) \hat{e}\left(\hat{\tau}_{1}, t\right) \cdots \hat{e}\left(\hat{\tau}_{q}, t\right) d \bar{\tau}_{1} \\
& \cdots d \bar{\tau}_{k} d \hat{\tau}_{1} \cdots d \hat{\tau}_{n}
\end{aligned}
$$

The latter can also be rewritten, by collecting from the summations in $k, n$ the terms independent of $k, n$ in the form:

$$
\begin{aligned}
i(t)= & F_{\mathrm{DC}}\{\bar{v}(t), \hat{v}(t)\}+\sum_{\substack{p=0 \\
(p, q) \neq(0,0)}}^{\infty} \sum_{\substack{q=0 \\
(p)}}^{t} \int_{t-\tau_{m}}^{(q)} \int_{t-\tau_{m}}^{t} \\
& \cdot \bar{e}\left(\bar{\tau}_{1}, t\right) \cdots \bar{e}\left(\bar{\tau}_{p}, t\right) \hat{e}\left(\hat{\tau}_{1}, t\right) \cdots \hat{e}\left(\hat{\tau}_{q}, t\right) \\
& \cdot\left[\sum_{k=p}^{\infty} \sum_{n=q}^{\infty} \bar{v}^{(k-p)}(t)\left(\begin{array}{c}
k \\
p
\end{array}\right) \hat{v}^{(n-q)}(t)\left(\begin{array}{l}
n \\
q
\end{array}\right)\right. \\
& \cdot{ }^{(k-p)} \int_{t-\tau_{m}}^{t} \int_{t-\tau_{m}}^{(n-q)} h_{k n}\left(t-\bar{\tau}_{1}, \cdots, t-\bar{\tau}_{k} ;\right. \\
& \left.t-\hat{\tau}_{1}, \cdots, t-\hat{\tau}_{n}\right) d \bar{\tau}_{p+1} \cdots d \bar{\tau}_{k} d \hat{\tau}_{q+1} \\
& \left.\cdots d \hat{\tau}_{n}\right] d \bar{\tau}_{1} \cdots d \bar{\tau}_{p} d \hat{\tau}_{1} \cdots d \hat{\tau}_{q}
\end{aligned}
$$

By defining $G_{p q}$ as the terms inside the square brackets in (22), the same equation can be written as

$$
\begin{aligned}
i(t)= & F_{\mathrm{DC}}\{\bar{v}(t) ; \hat{v}(t)\}+\sum_{\substack{p=0 \\
(p, q) \neq(0,0)}}^{\infty} \sum_{q=0}^{\infty} \int_{t-\tau_{m}}^{t} \int_{t-\tau_{m}}^{(q)} t \\
& \cdot G_{p q}\left\{\bar{v}(t), \hat{v}(t) ; t-\bar{\tau}_{1}, \cdots, t-\bar{\tau}_{p}\right. \\
& \left.t-\hat{\tau}_{1}, \cdots, t-\hat{\tau}_{q}\right\} \\
& \cdot \bar{e}\left(\bar{\tau}_{1}, t\right) \cdots \bar{e}\left(\bar{\tau}_{p}, t\right) \hat{e}\left(\hat{\tau}_{1}, t\right) \\
& \cdots \hat{e}\left(\hat{\tau}_{q}, t\right) d \bar{\tau}_{1} \cdots d \bar{\tau}_{p} d \hat{\tau}_{1} \cdots d \hat{\tau}_{q}
\end{aligned}
$$

which represents the Bivariate Nonlinear Integral Series.

Under the hypothesis that the dynamic deviations $\bar{e}, \hat{e}$ are small enough to make the integrals of dimension greater than one negligible in (23) (see Section II), the Bivariate Nonlinear Integral Model is obtained:

$$
\begin{aligned}
i(t)= & F_{\mathrm{DC}}\{\bar{v}(t), \hat{v}(t)\}+\int_{t-\tau_{m}}^{t}\left[G_{10}\{\bar{v}(t), \hat{v}(t) ; t-\tau\}\right. \\
& \left.\cdot \bar{e}(\tau, t)+G_{0 !}\{\bar{v}(t), \hat{v}(t) ; t-\tau\} \hat{e}(\tau, t)\right] d \tau
\end{aligned}
$$


As has been stated in Section II, (24) could also be obtained by means of a simple vectorial extension of the one-dimensional Nonlinear Integral Model (6).

\section{REFERENCES}

[1] R. Goyal, Ed., Monolithic Microwave Integrated Circuits: Technology \& Design. Norwood, MA: Artech House, 1989.

[2] P. H. Ladbrooke, MMIC Design: GaAs FETs and HEMTs. Norwood, MA: Artech House, 1989.

[3] D. Haigh and J. Everard, Eds., GaAs Technology and Its Impact on Circuits and Systems. London: Peregrinus, 1989.

[4] Special Issue on Multifunction MMIC's and their System Applications," IEEE Trans. Microwave Theory Tech., vol. 38, no. 9, Sept. 1990.

[5] V. A. Monaco and P. Tiberio, "Computer aided analysis of microwave circuits," IEEE Trans. Microwave Theory Tech., vol. MTT 22, no. 3, pp. 264-269, Mar. 1974

[6] F. Filicori, V. A. Monaco, and C. Naldi, "Simulation and design of microwave class-C transistor amplifiers through harmonic analysis." IEEE Trans. Microwave Theory Tech., vol. MTT-27, Dec. 1979.

[7] K. S Kundert and A. Sangiovanni-Vincentelli, "Simulation of nonlinear circuits in the frequency domain," IEEE Trans. ComputerAided Design, vol. CAD-5, no. 4, pp. 521-535, Oct. 1986

[8] F. Filicori and V. A. Monaco, "Computer-aided design of nonlinear microwave circuits,' Alta Frequenza, vol. LVII, no. 7, pp. 355-378, Sept. 1988.

[9] R. H. Jansen, R. G. Amold, and I. G. Eddison, "A comprehensive CAD approach to the design of MMIC's up to mm- wave frequencies," IEEE Trans. Microwave Theory Tech., vol. MTT-36, no. 2, pp. 208-219, Feb. 1988.

[10] J. W. Bandler and S. H. Chen, "Circuit optimization: the state of the art," IEEE Trans Microwave Theory Tech., vol. 36, no. 2, pp. 424443, Feb. 1988

[11] G. W. Rhyne, M. B. Steer, and B. D. Bates, "Frequency-domain nonlinear circuit analysis using generalized power series," IEEE Trans. Microwave Theory Tech., vol. MTT-36, no. 2, pp. 379-387, Feb. 1988.

[12] R. H. Jansen and L. Wiemer, "Full-wave theory based development of mm-wave circuit models for microstrip open and gap, step, bend and tee," in 1989 IEEE MTT-S Microwave Symp. Dig., vol. 2, Long Beach, CA, 1989, pp. 779-782.

[13] Special Issue: State-of-the-Art in Microwave CAD, Part I, Int. J. Microwave and Millimeter-Wave Computer-Aided Engineering, vol. 1, no. 1 , Jan. 1991.

[14] Speical Issue: State-of-the-Art in Microwave CAD, Part II, Int. J. Microwave and Millimeter-Wave Computer-Aided Engineering, vol. 1, no. 2, Apr. 1991

[15] F. Filicori, V. A. Monaco, and G. Vannini, "Computationally efficient multitone analysis of nonlinear microwave circuits," in Proc. 21st European Microwave Conf., Stuttgart, Sept. 1991, pp. 15501555.

[16] M. B. Steer, "Simulation of nonlinear microwave circuits-an historical perspective and comparisons," in 1991 IEEE MTT-S Microwave Symp Dig., vol. 2, Boston, 1991, pp. 599-602.

[17] J. B. Yan, R. J. Trew, and D. E. Stoneking "GaAs power MESFET performance sensitivity to profile and process parameter variations," in 1988 IEEE MTT-S Microwave Symp. Dig., vol. 1, New York, June 1988, pp. 343-346.

[18] J. W. Bandler, Q. Cai, R. M. Biernacki, S. H. Chen, and Q. J. Zhang, "Physics-based design and yield optimization of MMIC's," in Proc. 21st European Microwave Conf., Stuttgart, Sept. 1991, pp. 1515 1520.

[19] C. M. Snowden, Semiconductor Device Modelling. London: Peregrinus, 1988

[20] G. Ghione, C. Naidi, F. Filicori, M. Cippelletti, and G. P. Locatelli, "MESS: A two-dimensional physical device simulator and its application to the development of C-band power GaAs MESFET's." Alta Frequenza, vol. LVII, no. 7, pp. 295-309, Sept. 1988

[21] G. Ghione, F. Filicori, and C. Naldi, "Physical modeling of GaAs MESFET's in an integrated CAD environment: from device technology to microwave circuit performance," IEEE Trans. Microwave Theory Tech., vol. 37, no. 3, pp. 457-468, Mar. 1989.

[22] C. Jacoboni and P. Lugli, The Monte Carlo Simulation of Semiconductor Devices. Wien: Springer Verlag, 1989.
[23] C. M. Snowden, "A large-signal physical MESFET model for computer-aided design and its applications," IEEE Trans. Microwave Theory Tech., vol. MTT-37, no. 12, pp. 2039-2045, Dec. 1989.

[24] J. W. Bandler, Q. J. Zhang, and Q. Cai, "Nonlinear circuit optimization with dynamically integrated physical device models,"' in 1990 IEEE MTT-S Microwave Theory Dig., vol. 1, pp. 303, 306, Dallas, June 1990.

[25] "Focus on advanced topics in physical III-V device simulation," $E u$ ropean Trans. Telecommunications, vol. 1, no. 4, July 1990.

[26] C. Rauscher and H. Willing, "Simulation of nonlinear microwave FET performance using a quasi-static model," IEEE Trans. Microwave Theory Tech., vol. MTT-27, no. 10, pp. 834-840, Oct. 1979.

[27] G. P. Bava, S. Benedetto, E. Biglieri, C. Naldi, U. Pisani, V. Pozzolo, F. Filicori, and V. A. Monaco, "Modelling and performance simulation techniques of GaAs MESFET's for microwave power amplifiers," ESA/ESTEC Report, contract 4043/79, Feb. 1982.

[28] M. Sango et al., "A GaAs MESFET large-signal circuit model for nonlinear analysis," in 1988 IEEE MTT-S Microwave Symp. Dig. New York, 1988, pp. 1053-1056.

129] J. W. Bandler, Q. J. Zhang, S. Ye, and S. H. Chen, "Efficient largesignal FET parameter extraction using harmonics," IEEE Trans. Microwave Theory Tech., vol. 37, no. 12, pp. 2099-2108, Dec. 1989.

[30] A. Madjar and F. J. Rosenbaum, " A large-signal model for the GaAs MESFET," IEEE Trans. Microwave Theory Tech., vol. MTT-29, no. 8, pp. 781-788, Aug. 1981

[31] M. A. Khatibzadeh and R. J. Trew, "A large-signal, analytic model for the GaAs MESFET," IEEE Trans. Microwave Theory Tech., vol. 36, no. 2, pp. 231-238, Feb. 1988.

[32] F. Filicori, V. A. Monaco, and G. Vannini, "Mathematical approaches to electron device modelling for nonlinear microwave circuit design: state of the art and present trends," Invited Paper, European Trans. Telecommunications, vol. I, no. 6, pp. 641-654, Nov. 1990.

[33] F. Filicori and G. Vannini, "Mathematical approach to large-signal modelling of electron devices," Electron. Lett., vol. 27, no. 4, pp. 357-359, Feb. 1991.

[34] V. Volterra, Theory of Functionals and of Integral and Integro-differential Equations. New York: Dover, 1959.

[35] J. Bussgang, L. Ehrman, and J. Graham, "Analysis of nonlinear systems with multiple inputs," Proc. IEEE, vol. 62, no. 8, pp. 10881119. Aug. 1974.

[36] S. A. Maas. Nonlinear Microwave Circuits. Norwood, MA: Artech House, 1988

[37] C. L. Law and C. S. Aitchison, "Prediction of wide-band power performance of MESFET distributed amplifiers using the Volterra series representation," IEEE Trans. Microwave Theory Tech., vol. MTT34, no. 12, pp. 1308-1317, Dec. 1986.

[38] P. J. Lunsford and M. B. Steer, “The relationship between bivariate Volterra analysis and power series analysis with application to the behavioral modeling of microwave circuits," Int. J. Microwave and Millimeter-Wave Computer-Aided Engineering, vol, 1, no. 3, pp. 253-262, July 1991.

[39] S. Boyd, Y. S. Tang, and L. O Chua, "Measuring Volterra kernels," IEEE Trans. Circuits Syst, vol. CAS-30, no. 8, pp. 571-577. Aug. 1983.

[40] L. O. Chua and Y. Liao, "Measuring Volterra kernels (II)," Int. J. Circuit Theory and Applications, vol. 17, pp. 151-190, 1989.

[41] F. Filicori, G. Vannini, A. Mediavilla, and A. Tazon, "Modelling low-frequency dispersion phenomena in GaAs MESFETs," to be published.

[42] F. Filicori and G. Ghione, "A computationally efficient unified approach to the sensitivity and noise numerical analysis of semiconductor devices," IEEE Trans. Computer-Aided Design, to be published.

Fabio Filicori, for a photograph and biography, see this issue, p. 1352.

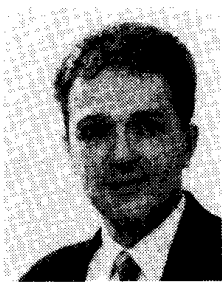

Giorgio Vannini (S'87) was born in Città di Castello, Italy, in 1960 . He received the degree in electronic engineering from the University of Bologna in 1986

Since then he has been with the Dipartimento di Elettronica, Informatica e Sistemistica of the University of Bologna where he has completed a Ph.D. course on Electronic Engineering and Computer Sciences. In 1992 he joined the Dipartimento di Elettronica, Informatica e Sistemistica of the University of Bologna as a Researcher. His 
research activity is mainly oriented to electron device modeling, CAD techniques for Microwave Integrated Circuits and Nonlinear circuit analysis and design.

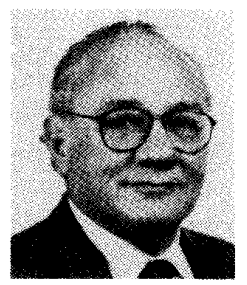

Vito Antonio Monaco (M'67-SM'87) was born in Brindisi, Italy, in 1932. He received the degree in electrical engineering from the University of Bologna in 1957. From 1958 he was with the School of Engineering, University of Bologna where he was an Assistant Professor of Electrical Communications until 1961, when he became an Associate Professor of Radio and Radar Systems and, in 1967, of Applied Electronics.

Since 1970 he has been Full Professor of Applied Electronics with the Dipartimento di Elettronica, Informatica e Sistemistica of the University of Bologna. His research activity concerned the field of radio electronics and computer aided design of microwave circuits. In particular he proposed the adoption of sparse matrix techniques in algorithms for the analysis of microwave circuits. The computation of network function sensitivities in terms of circuit parameters and the evaluation of manufacturing yield of microwave circuits have also been topics of his research. More recently his research activity has regarded the adoption of harmonic balance techniques in the design of nonlinear microwave circuits and nonlinear modeling of electron devices for microwave applications.

For his research activity in computer aided design of microwave circuits Prof. Monaco was awarded with the Gold Medal "AEI Pubblicazioni 1990." He has been Chairman of the Electronic Department of the University of Bologna (from 1975 to 1978) and Chairman of the North-Italy Section of IEEE (from 1987 to 1990). Presently he is member of the Advisory Committee on Engineering and Architecture and on Science and Technology of Information of the National Research Council in Italy.

$\mathrm{Mr}$. Monaco is Socio Benemerito of AEI-Italian Electrical and Electronic Association; President of the Gruppo Specialistico CCTE-Circuiti Componenti e Tecnologie Elettroniche of AEI; member of the Editorial Board of Alta Frequenza and of ETT-European Transactions on Telecommunications and related technologies. 